\title{
Una aproximación con modelos competitivos a la dismorfia muscular: El papel de la autoestima y la regulación emocional
}

\author{
Laura GALIANA, Laura BADENES-RIBERA y María C. FUENTES
}

\author{
Departamento de Metodología de las Ciencias del Comportamiento, Universitat de València
}

(Recibido, 10 de Junio de 2018; Aceptado, 24 de Septiembre de 2018)

RESUMEN: Introducción: En la literatura sobre dismorfia muscular (DM) dos variables se han mostrado clave en su predicción: la autoestima y la regulación emocional. No obstante, los estudios que consideran ambas variables son escasos. El objetivo de este estudio es evaluar la relación entre dichas variables y la $\mathrm{DM}$, explorando distintos acercamientos teóricos o modelos: 1) una relación directa entre DM y regulación emocional, e indirecta entre DM y autoestima, mediada por la regulación emocional; 2) una relación directa entre DM y regulación emocional, y una relación tanto directa entre DM y autoestima, como mediada por la regulación emocional; 3) una relación directa entre DM y autoestima, y una relación indirecta entre DM y regulación emocional; y 4) una relación directa entre DM y autoestima, y una relación tanto directa como indirecta, a través de autoestima, entre DM y regulación emocional. Método: Participaron 251 estudiantes universitarios (72.7\% mujeres) con una media de edad de 21.31 años (DT $=$ 2.52). Resultados: De los modelos probados, el modelo 2 mostró el mejor ajuste: $\chi 2=112.605, \mathrm{gl}=42, \mathrm{p}$ $<.001$; CFI $=.916$; $\mathrm{RMSEA}=.082(\mathrm{IC}=.064, .101) ; \mathrm{SRMR}=.064 ; \mathrm{AIC}=5484.284$; reteniéndose como mejor representación de los datos. Conclusiones: La autoestima y la regulación emocional, pese a ser predictores de la DM, muestran distintas relaciones con ella, pues mientras que la autoestima la predice tanto directamente como a través de la regulación emocional, con gran capacidad explicativa, la regulación emocional mantiene solo una relación directa con la DM y de menor tamaño.

Palabras clave: dismorfia muscular, autoestima, regulación emocional, modelos de ecuaciones estructurales, estudiantes universitarios.

\section{A Competitive Model Approach to Muscular Dysmorphia: The Role of Self-Esteem and Emotional Regulation}

\begin{abstract}
Background: Among muscular dysmorphia (MD) literature, two variables have become key in its prediction: self-esteem and emotional regulation. However, studies considering both variables at a time are scarce. The aim of current research is to assess the relation among these variables and MD, exploring different theoretical approaches or models: 1) a direct relation between MD and emotional regulation, and an indirect one between $\mathrm{MD}$ and self-esteem, mediated by the emotional regulation; 2) a direct relation between MD and emotional regulation, and both a direct and an indirect relation with selfesteem, mediated by emotional regulation; 3) a direct relation between MD and self-esteem, and an indirect one between MD and emotional regulation, mediated by self-esteem; and 4) a direct relation between MD and self-esteem, and both a direct and an indirect relation between MD and emotional
\end{abstract}


regulation, mediated by self-esteem. Methods: 251 university students participated in the study $(72.7 \%$ were women). Mean age was 21.31 years old ( $\mathrm{SD}=2.52)$. Results: Among the tested models, model 2 showed better fit: $\chi^{2}=112.605, \mathrm{df}=42, p<.001 ; \mathrm{CFI}=.916$; $\mathrm{RMSEA}=.082(\mathrm{IC}=.064, .101)$; $\mathrm{SRMR}=$ .064 ; $\mathrm{AIC}=5484.284$; and, thus, it was retained as the best representation of the data. Conclusions: Selfesteem and emotional regulation, although both being predictors of MD, showed different relations with it: whereas self-esteem predicted direct and indirectly MD, mediated by emotional regulation, with great predictive power, emotional regulation only showed a direct relation, of smaller size.

Keywords: muscular dysmorphia, self-esteem, emotional regulation, structural equation modeling, university students.

Correspondencia: Laura Galiana, Departamento de Ciencias del Comportamiento, Universitat de València, Av. Blasco Ibañez, 21, 46010, Valencia (España). E-mail: laura.galiana@uv.es

\section{Introducción}

Las preocupaciones sobre el aspecto corporal en hombres y mujeres son habituales en las sociedades occidentales, fruto de un énfasis sobre la imagen corporal cada vez mayor (Bartsch, 2007; Griffiths et al., 2016). En este contexto, se ha descrito un nuevo fenómeno que puede afectar a aquellas personas con alteraciones de la imagen corporal: la dismorfia muscular (DM en adelante).

La DM es un trastorno de la imagen corporal, que tradicionalmente se presenta en varones que se perciben con poca muscularidad y muestran una creciente preocupación por aumentar su masa muscular hasta conseguir una forma del cuerpo ideal (Galiana, BadenesRibera y Fuentes, 2017; Grieve, 2007; Grieve y Shacklette, 2012; Grieve, Truba y Bowersox, 2009; Olivardia, 2001; Pope, Gruber, Choi, Olivardia y Phillips, 1997). Los primeros en identificar este trastorno fueron Pope, Katz y Hudson (1993), en un estudio sobre los efectos psiquiátricos del uso de esteroides en culturistas, en el que encontraron como algunos culturistas mostraban distorsiones en su imagen corporal, percibiéndose más pequeños y débiles de lo que eran en realidad. Actualmente, existe consenso en considerar la DM como un trastorno que se caracteriza tanto por síntomas cognitivos como conductuales, incluyendo una marcada preocupación sobre el propio físico por no ser suficientemente musculoso, a pesar de estar por encima de la media en cuanto a musculación, y un exceso de ejercicio y levantamiento de pesas, cuya imposibilidad de realizar da lugar a ansiedad, creando consecuentemente problemas de funcionamiento laboral y social (Murray, Rieger, Touyz y De la Garza, 2010).

Así pues, la DM es similar a los trastornos de la conducta alimentaria en la medida en que ambos conllevan una preocupación por los defectos del cuerpo y el uso de varias técnicas para intentar cambiarlos (Galiana et al., 2017). Siendo así, un amplio cuerpo de literatura se ha centrado en analizar la DM y la psicopatología relacionada con la ingesta, la forma y el peso de los hombres desde la óptica de los trastornos de la alimentación (Murray, Rieger, Karlov y Touyz, 2013). Partiendo del hecho de que trastornos como la anorexia nerviosa y la DM comparten niveles similares de restricción dietética, preocupaciones sobre el peso y la forma, ejercicio compulsivo y deterioro funcional, se han sugerido similitudes sintomatológicas de ambos trastornos (Murray et al., 2013). Esta misma literatura ha apuntado, además, mismas causas o patrones causantes, con niveles similares en variables 
como el perfeccionismo, obsesiones, anhedonia, autoestima o desregulación emocional (Davis y Scott-Robertson, 2000; Lamanna, Grieve, Derryberry, Hakman y McClure, 2010; Murray et al., 2013).

En esta línea, los estudios con fines etiológicos que han estudiado el desarrollo de psicopatologías relacionadas con los trastornos de la imagen corporal han puesto el foco en la relación con diversas variables, siendo recurrente entre ellos la aparición de un patrón de relación negativo entre niveles de autoestima y puntuaciones en DM (Grieve, 2007; Murray et al., 2013; Olivardia, 2001). La literatura consultada apunta a que altos niveles de autoestima, en cuanto al desarrollo de la DM se refiere, podrían funcionar como barreras protectoras frente al desarrollo del trastorno, tal y como se ha visto previamente en la literatura sobre trastornos de la alimentación (Joshi, Herman y Polivy, 2004; Murray et al., 2013). En el campo de los trastornos de la imagen corporal, bajos niveles de autoestima se han observado en varones con síntomas de DM, como por ejemplo, falta de satisfacción con la muscularidad o ansiedad por el físico (Cafri, Strauss y Thompson, 2002; Grieve, Jackson, Reece, Marklin y Delaney, 2008; Olivardia, Pope, , Borowiecki y Cohane, 2004; Wolke y Sapouna, 2008).

Otra variable que recientemente se ha relacionado con el desarrollo de alteraciones en la imagen corporal y DM ha sido la desregulación emocional (Cunningham, Griffiths, Baillie y Murray, 2018; Griffiths, Angus, Murray y Touyz, 2014; Murray et al., 2013). Entendida como la incapacidad para identificar, comprender y aceptar experiencias emocionales, controlar comportamientos impulsivos en momentos de estrés, y poseer flexibilidad para adaptar las respuestas emocionales a las características de la situación (Gratz y Roemer, 2004). La regulación emocional sería un factor clave para el desarrollo de comportamientos desadaptativos como los implicados en la DM (i.e., ejercicio efectivo) (Lavender y Anderson, 2010). Tal y como ya ocurría con la autoestima, el papel de la (des)regulación emocional se había apuntado previamente en estudios centrados en trastornos de la alimentación (Killen et al., 1996; Sim y Zeman, 2005, 2006).

Parece claro, pues, que tanto la autoestima como la regulación emocional juegan un papel importante en el desarrollo de la DM. No lo es tanto, sin embargo, qué papel es ese. En el contexto de los trastornos de la alimentación, algunos autores han propuesto que la regulación emocional podría afectar a los trastornos alimentarios indirectamente, a través de su efecto sobre la autoimagen (Monell, Högdahl, Mantilla y Birgegård, 2015). Según esta línea de investigación, los problemas de regulación emocional implicarían una autoimagen negativa, que a su vez tendría como consecuencia el desarrollo de trastornos de la imagen corporal. Así expuesto, podría pensarse que también la autoestima podría jugar este papel mediador entre la regulación emocional y la DM, no existiendo, por tanto, el efecto directo entre estas dos variables. Otros autores, sin embargo, apuntan a que la autoestima podría no tener un efecto directo sobre la DM, sino que lo haría a través de su efecto sobre el afecto negativo, (Grieve, 2007), afecto que tradicionalmente se ha relacionado con la desregulación emocional (para revisiones sobre el tema, consultar Dubey, Pandey y Mishra, 2010, o Taylor, 2000). Según esta otra hipótesis, la autoestima sería la causante del afecto negativo y la desregulación emocional que lo acompaña, y esta desregulación sería la que causaría la DM. A pesar de estos desarrollos teóricos, la evidencia empírica recogida se ha limitado, bien a otros trastornos de la imagen corporal, bien a otras variables, pero ningún 
estudio, hasta donde sabemos, se ha centrado en aportar evidencia sobre los efectos directos e indirectos que autoestima y regulación emocional tienen conjuntamente en la predicción de la DM.

\section{Objetivo del estudio}

Teniendo en cuenta lo hasta aquí expuesto, el objetivo de este estudio es evaluar el papel en el desarrollo de DM que juegan la autoestima y la regulación emocional, explorando para ello distintos acercamientos teóricos o modelos competitivos, que pueden consultarse en la Figura 1:

1. El primer modelo o Modelo 1 pone a prueba la existencia de una relación directa entre DM y regulación emocional, e indirecta entre DM y autoestima, mediada por la regulación emocional.

2. El Modelo 2 hipotetiza una relación directa entre DM y regulación emocional, y una relación tanto directa entre DM y autoestima, como mediada por la regulación emocional.

3. El Modelo 3 especifica una relación directa entre DM y autoestima, y una relación indirecta entre DM y regulación emocional.

En el Modelo 4 se pone a prueba una relación directa entre DM y autoestima, y una relación tanto directa como indirecta, a través de autoestima, entre DM y regulación emocional. 
Figura 1. Modelos puestos a prueba.

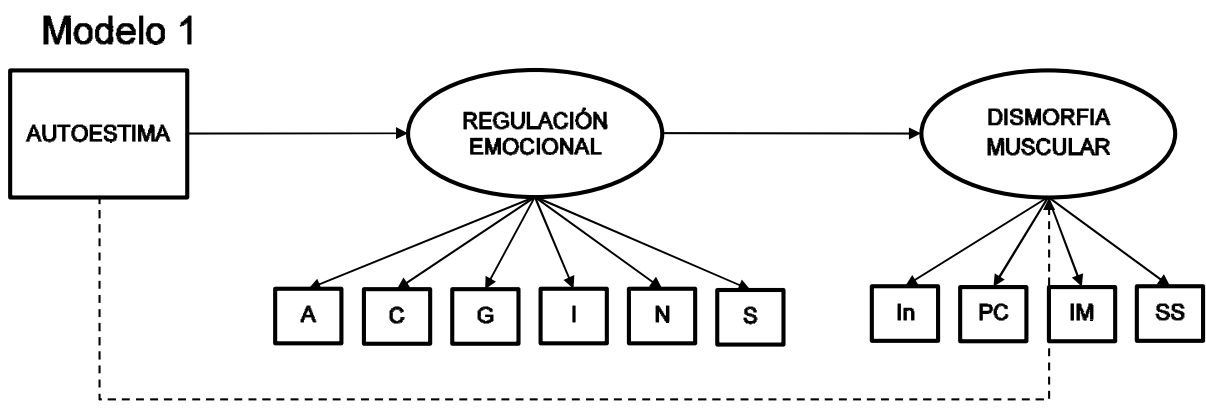

\section{Modelo 2}

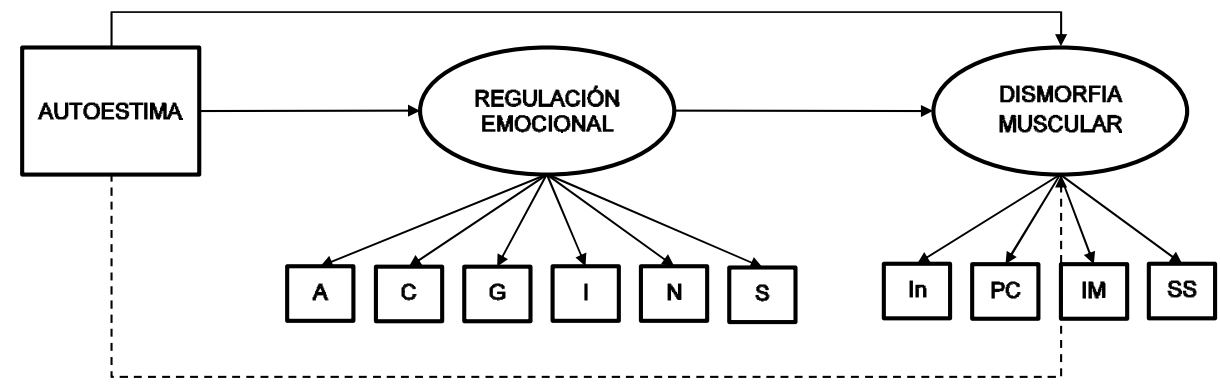

\section{Modelo 3}

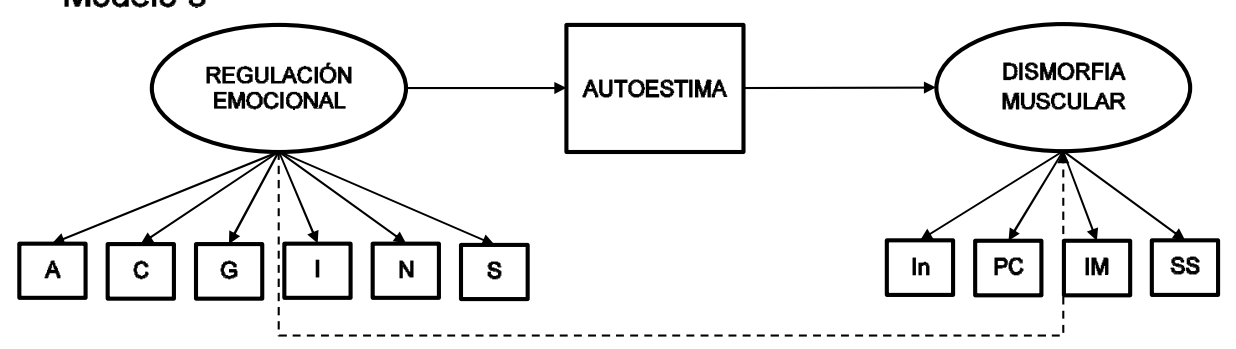

Modelo 4

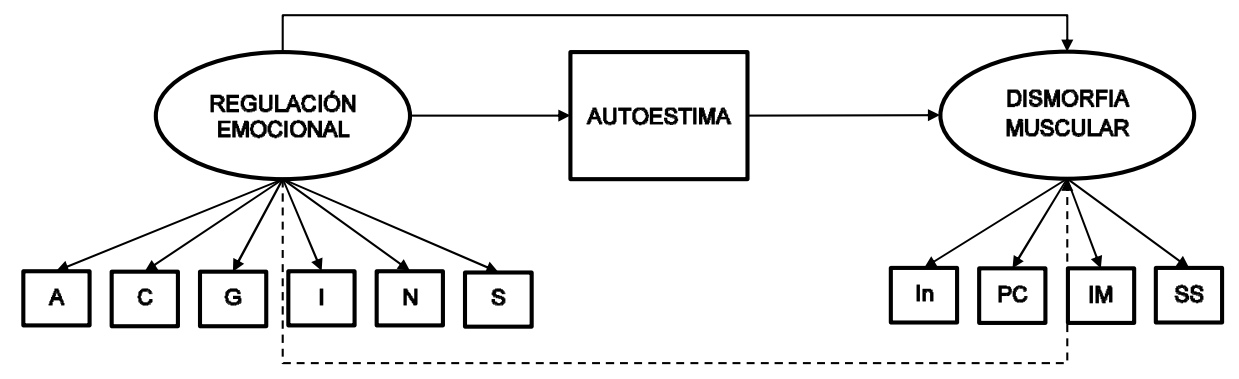

Notas: $\mathrm{A}=$ autoconciencia, $\mathrm{C}=$ claridad, $\mathrm{G}=$ metas, $\mathrm{I}=$ impulso, $\mathrm{N}=$ no-aceptación, $\mathrm{S}=$ estrategias, $\mathrm{In}=$ inadecuación, $\mathrm{PC}=$ persistencia y compulsividad, $\mathrm{IM}=$ incremento de la musculatura, $\mathrm{SS}=$ sacrificio social. 


\section{Método}

\section{Participantes}

Participaron en el estudio 251 estudiantes universitarios de Psicología de la ciudad de Valencia. $72.7 \%$ mujeres y el $27.3 \%$ restante fueron hombres. La media de edad fue de 21.31 años $(D T=2.52)$, oscilando entre los 18 y 29 años.

\section{Instrumentos}

Además de características sociodemográficas, se recogió información sobre dismorfia muscular y diversas variables relacionadas. En concreto, para este estudio se utilizaron los siguientes instrumentos.

Inventario de Dismorfia Muscular-Revisado (Muscle Dysmorphia Inventory, MDI-R; Short, 2005). Se utilizó la versión validada en español por Badenes-Ribera, Fuentes y Galiana (2017), en la que se evalúan cuatro dimensiones: inadecuación, persistencia y compulsividad, incremento de la musculatura y sacrificio social. Los ítems puntúan en una escala tipo Likert que oscila entre 1 (totalmente en desacuerdo) y 6 (totalmente de acuerdo). La fiabilidad para esta muestra fue de .867 (nadecuación), .848 (persistencia y compulsividad), .790 (incremento de la musculatura) y .751 (sacrificio social).

Escala de Autoestima de Rosenberg (Rosenberg Self-Esteem Scale, RSES; Rosenberg, 1965). Esta escala es un cuestionario de autoinforme que evalúa una única dimensión de autoestima global. Los ítems puntúan en una escala tipo Likert de 1 (totalmente en desacuerdo) a 4 (totalmente de acuerdo). La fiabilidad en esta muestra fue de .853 .

Escala de Dificultades en la Regulación Emocional (Difficulties with Emotional Regulation Scale, DERS; Gratz y Roemer, 2004). Es un instrumento diseñado para medir la desregulación emocional. La escala de autoinforme consiste en 18 ítems en su versión abreviada, reunidos en seis factores: no aceptación de respuestas emocionales (no-aceptación), dificultades en conductas dirigidas a metas cuando se está alterado (metas), dificultades en controlar conductas impulsivas cuando se está alterado (impulsividad), acceso limitado a estrategias de regulación emocional percibidas como efectivas (estrategias), falta de consciencia emocional (autoconciencia), y falta de claridad emocional (claridad). En este estudio, la fiabilidad fue de .871 .

\section{Procedimientos}

La encuesta se llevó a cabo después de las clases y tuvo una duración aproximada de 20 minutos. La participación fue voluntaria y anónima, con una tasa de participación aproximada del 70\%. Durante la cumplimentación de la encuesta estuvieron presentes investigadores entrenados para ofrecer indicaciones y resolver dudas.

Todos los procedimientos se desarrollaron siguiendo los estándares éticos y de acuerdo a la declaración de Helsinki (1964) y sus posteriores modificaciones. 


\section{Análisis de datos}

Los análisis de datos incluyeron estadísticos descriptivos de las variables objeto de estudio y la puesta a prueba de diversos modelos de ecuaciones estructurales competitivos. Estos modelos pusieron a prueba diversos efectos entre los tres constructos de interés (DM, autoestima y regulación emocional) y fueron estimados con Mplus (Muthén y Muthén, 1998-2011).

El ajuste de los modelos se evaluó mediante diversos índices de ajuste, que incluyeron: el estadístico chi-cuadrado (Kline, 1998; Ullman, 1996); el CFI, el RMSEA y el SRMR. Valores de CFI mayores de .90 y de RMSEA y SRMR menores de .08 se consideran indicativos de buen ajuste del modelo hipotetizado a los datos ( $\mathrm{Hu}$ y Bentler, 1999). Adicionalmente, y para comparar los modelos, se calcularon diferencias de CFI y se utilizó el criterio de información de Akaike (Akaike's information criterion, AIC). Las diferencias de CFI se consideran relevantes a partir de 0.05 (Little, 1997) o 0.01 (Cheung y Rensvold, 2002), según los autores consultados. El AIC, por su parte, se considera óptimo cuando su valor es menor.

\section{Resultados}

Los estadísticos de las variables objeto de estudio pueden consultarse en la Tabla 1.

Tabla 1. Estadísticos descriptivos de las variables objeto de estudio

\begin{tabular}{lcccc}
\hline & $M$ & $D T$ & Mínimo & Máximo \\
\hline Autoestima & 3.34 & 0.55 & 1.50 & 4.00 \\
Autoconciencia & 3.98 & 0.71 & 1.33 & 5.00 \\
Claridad & 2.24 & 0.90 & 1.00 & 5.00 \\
Metas & 2.58 & 0.83 & 1.00 & 5.00 \\
Impulso & 2.34 & 0.84 & 1.00 & 5.00 \\
No-aceptación & 2.69 & 0.89 & 1.00 & 5.00 \\
Estrategias & 2.59 & 0.88 & 1.00 & 5.00 \\
Inadecuación & 2.35 & 1.19 & 1.00 & 6.00 \\
Persistencia y compulsividad & 1.61 & 0.97 & 1.00 & 5.50 \\
Incremento de la musculatura & 2.79 & 1.24 & 1.00 & 6.00 \\
Sacrificio social & 1.14 & 0.42 & 1.00 & 4.33 \\
\hline
\end{tabular}

Notas: $M=$ media; $D T=$ desviación típica; Mínimo = puntuación mínima; Máximo = puntuación máxima.

En cuanto a los modelos, se estimaron cuatro modelos, que planteaban relaciones de mediación total y de mediación parcial entre autoestima, regulación emocional y DM. Tal y como puede observarse en la Tabla 2, los Modelos 2, 3 y 4 mostraron un ajuste adecuado. Se descartó, por tanto, el Modelo 1 como una representación adecuada de los datos. Cuando se calcularon las diferencias de CFI de los Modelos 2, 3 y 4, los Modelo 2 y 4 mostraron idéntico CFI, y superiores en 0.05 al Modelo 3 y en 0.39 al Modelo 1. Las diferencias en CFI entre dichos modelos superan tanto el punto de corte propuesto por Cheung y Rensvold (2002), como el más laxo de Little (1997). Cuando se tuvo en cuenta el AIC, el Modelo 2 fue el que 
obtuvo un AIC de menor valor, indicando así una mejor compensación entre ajuste y simplicidad. El Modelo 2 se retuvo, por tanto, como mejor representación de los datos.

Tabla 2. Índices de ajuste de los modelos puestos a prueba

\begin{tabular}{lccccccc}
\hline & $\chi^{2}$ & $\mathrm{gl}$ & $p$ & CFI & RMSEA [IC 95\%] & SRMR & AIC \\
\hline Modelo 1 & 146.201 & 43 & $<.001$ & .877 & $.098[.081, .116]$ & .081 & 5519.641 \\
Modelo 2 & 112.605 & 42 & $<.001$ & .916 & $.082[.064, .101]$ & .064 & 5484.284 \\
Modelo 3 & 117.812 & 43 & $<.001$ & .911 & $.083[.065, .101]$ & .073 & 5937.921 \\
Modelo 4 & 113.026 & 42 & $<.001$ & .916 & $.082[.064, .101]$ & .063 & 5933.648 \\
\hline
\end{tabular}

Nota : $\mathrm{gl}=$ grados de libertad; IC = intervalo de confianza. El resto de índices han sido explicados en el apartado Análisis de datos.

Las estimaciones de los parámetros del modelo retenido, que pueden observarse en la Figura 2, apuntan un efecto estadísticamente significativo tanto directo como indirecto de la autoestima sobre la DM, y un efecto directo estadísticamente significativo y negativo de la regulación emocional sobre este trastorno. Así pues, mayores niveles de autoestima se relacionan con menores puntuaciones en dismorfia muscular y también con mayor regulación emocional. Estos niveles de regulación emocional, a su vez, se relacionan de forma negativa con la DM (a mayor regulación emocional, menor puntuación en dismorfia muscular). El modelo consiguió explicar el 19.6\% de la regulación emocional y el 49.0\% de la DM.

Figura 2. Modelo estructural que estudia la relación de la autoestima, la regulación emocional y la DM.

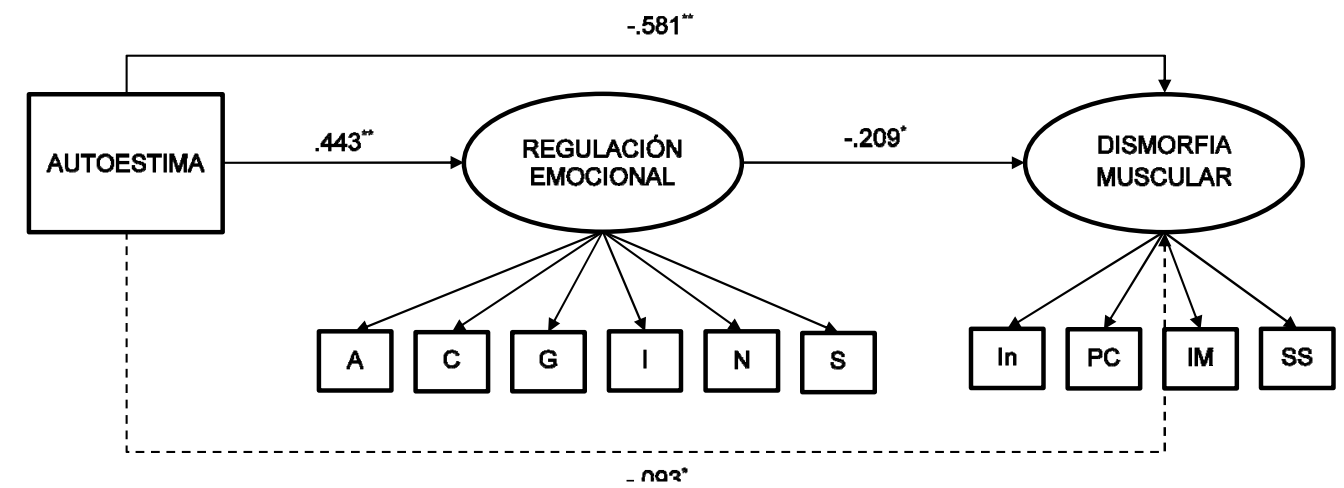

Notas: Todos los coeficientes de regresión presentados son estandarizados.

Todas las saturaciones factoriales resultaron estadísticamente significativas.

$* p<.05, * * p<.01$.

$\mathrm{A}=$ autoconciencia, $\mathrm{C}=$ claridad, $\mathrm{G}=$ metas, $\mathrm{I}=$ impulso, $\mathrm{N}=$ no-aceptación, $\mathrm{S}=$ estrategias, $\mathrm{In}=$ inadecuación, $\mathrm{PC}=$ persistencia y compulsividad, $\mathrm{IM}=$ incremento de la musculatura, $\mathrm{SS}=$ sacrificio social.

\section{Discusión}

El objetivo de este estudio era evaluar el papel que juegan la autoestima y la regulación emocional en el desarrollo de DM, explorando cuatro acercamientos teóricos o modelos: 1) una relación directa entre DM y regulación emocional, e indirecta entre DM y autoestima, mediada por la regulación emocional; 2) una relación directa entre DM y regulación emocional, y una 
relación tanto directa entre DM y autoestima, como mediada por la regulación emocional; 3) una relación directa entre DM y autoestima, y una relación indirecta entre DM y regulación emocional; y 4) una relación directa entre DM y autoestima, y una relación tanto directa como indirecta, a través de autoestima, entre DM y regulación emocional.

Los resultados de este trabajo apuntan a que es el Modelo 2, aquél en el que la relación entre autoestima y DM está parcialmente mediada por la regulación emocional, el que es una mejor representación los datos. Parece, pues, que ambas variables, autoestima y regulación emocional, tendrían una capacidad predictiva directa sobre la DM, pero la autoestima tendría, además, un papel indirecto a través de la regulación emocional. Es decir, la autoestima podría tener un importante rol en la etiología, no solo de la DM, sino también de la regulación emocional.

La evidencia aquí recogida va en la línea del modelo propuesto por Grieve (2007), que postulaba una relación indirecta entre autoestima y DM, mediada por el afecto negativo. Los presentes resultados apuntan a una mediación realizada por la desregulación emocional, desregulación que habitualmente cohabita con ese tipo de afecto. En este sentido, podría apuntarse que la autoestima crea un estado de vulnerabilidad emocional, que es, en último término, el responsable de los síntomas de DM. No obstante, el presente trabajo va un paso más allá, añadiendo a este efecto indirecto un efecto directo, aunque como se ha visto, de menor magnitud. La autoestima es, por tanto, clave en cuanto al desarrollo de un estado emocional previo al desarrollo de DM, pero también una variable a tener en cuenta en dicho desarrollo per se (Cafri et al., 2002; Grieve, 2007; Grieve et al., 2008; Murray et al., 2013; Olivardia, 2001; Olivardia et al., 2004; Wolke y Sapouna, 2008).

No se sostiene según la evidencia encontrada, sin embargo, la hipótesis basada en el trabajo de Monell et al. (2015). Estos autores planteaban que no es la falta de regulación emocional la que causa directamente la imagen corporal, sino que lo hace a través de su influencia sobre la auto-imagen: la incapacidad para identificar, comprender y aceptar experiencias emocionales y controlar comportamientos impulsivos repercutiría sobre las interacciones sociales que mantienen los individuos, produciendo unas interacciones sociales desadaptativas y, por tanto, influyendo de forma negativa sobre la auto-imagen. Este problema de auto-imagen sería, según la aproximación de Monell et al. (2015), la causante de los trastornos de este tipo. Siguiendo esta lógica, se puso a prueba la hipótesis de que este mismo modelo teórico funcionara con la autoestima, que podría verse disminuida por los problemas de regulación emocional, siendo esta disminución de la autoestima lo que produciría los trastornos de la imagen corporal, en este caso, la DM. El modelo así planteado, sin embargo, obtuvo un peor ajuste.

Los resultados aquí obtenidos son relevantes por diversas razones. Se trata del primer estudio que pone a prueba efectos directos e indirectos de autoestima y regulación emocional sobre la DM. Por otra, el haber encontrado evidencia clara sobre un modelo teórico que establece unas relaciones entre las variables objeto de estudio debiera permitir diseñar de forma más eficaz las intervenciones dirigidas no solo al tratamiento de la DM, sino también a su prevención. Los efectos tanto directos como indirectos de la autoestima sugieren esta variable como un objetivo importante para el tratamiento, teniendo mayor efecto directo, por comparación al efecto de la desregulación emocional. No obstante, no hay que desdeñar el rol que juega la regulación emocional en este trastorno, pues contribuye a su desarrollo o mantenimiento, explicándolo también de forma estadísticamente significativa. Futuras intervenciones para el tratamiento y la prevención de la DM deberán tomar en cuenta estas 
aportaciones, haciendo especial hincapié en el trabajo de la autoestima, que servirá de barrera protectora frente al trastorno y también frente a la desregulación emocional.

\section{Referencias}

Badenes-Ribera, L., Fuentes, M. C., y Galiana, L. (2017). Inventario de dismorfia muscular (DMI-R): Fiabilidad y validez en una muestra universitaria española. En J. C. Núñez, M. C. Pérez-Fuentes, M. M. Molero, J. J. Gázquez, A. Martos, A. B. Barragán y M. M. Simón (Comps.), Temas actuales de investigación en las áreas de la Salud y la Educación (pp. 1031-1045). España: Scinfoper.

Bartsch, D. (2007). Prevalence of body dysmorphic disorder symptoms and associated clinical features among Australian university students. Clinical Psychologist, 11, $16-23$. http://dx.doi.org/10.1080/13284200601178532

Cafri, G., Strauss, J., y Thompson, J.K. (2002). Male body image: Satisfaction and its relationship to well-being using the somatomorphic matrix. International Journal of Men's Health, 1, 215.

Cheung, G. W., y Rensvold, R. B. (2002). Evaluating goodness-of-fit indices for testing measurement invariance. Structural Equation Modeling, 9, 233-255.

Cunningham, M. L., Griffiths, S., Baillie, A., y Murray, S. B. (2018). Emotion Dysregulation Moderates the Link Between Perfectionism and Dysmorphic Appearance Concern. Psychology of Men \& Masculinity, 19(1), 59-68.

Davis, C., y Scott-Robertson, L. (2000). A psychological comparison of females with anorexia nervosa and competitive male bodybuilders: body shape ideals in the extreme. Eating Behaviors, 1(1), 33-45.

Dubey, A., Pandey, R., y Mishra, K. (2010). Role of Emotion Regulation Difficulties and Positive/Negative Affectivity in Explaining Alexithymia - Health Relationship: An overview. Indian Journal of Social Sciences Researches, 7(1), 20-31.

Galiana, L., Badenes-Ribera, L., y Fuentes, M. C. (2017). Validación de la versión española del Inventario del Trastorno por Dismorfia Muscular en una muestra de estudiantes universitarios. En J. C. Núñez, M. M. Molero, J. J. Gázquez, M. C. Pérez-Fuentes, M. M. Simón, A. Martos y A. B. Barragán (Comps.), Atención a las necesidades comunitarias para la Salud (pp. 41-45). España: Scinfoper.

Gratz, K., \& Roemer, L. (2004). Multidimensional assessment of emotion regulation and dysregulation: Development, factor structure, and initial validation of the difficulties in emotion regulation scale. Journal of Psychopathology and Behavioral Assessment, 26, 41-54. http://dx.doi.org/10.1023/B:JOBA.0000007455.08539.94

Grieve, F. G. (2007). A conceptual model of factors contributing to the development of muscle dysmorphia. Eating Disorders, 15(1), 63-80.

Grieve, F. G., \& Shacklette, M. D. (2012). Brief report on men's bodies and mood: Correlates between depressive symptoms and muscle dysmorphia symptoms. North American Journal of Psychology, 14, 563-568.

Grieve, F. G., Truba, N., \& Bowersox, S. (2009). Etiology, assessment, and treatment of muscle dysmorphia. Journal of Cognitive Psychotherapy: An International Quarterly, 23(4), 306-314. 
Grieve, F.G. Jackson, L., Reece, T., Marklin, L., y Delaney, A. (2008). Correlates of social physique anxiety in men. Journal of Sport Behavior, 31, 329-337.

Griffiths, S., Angus, D., Murray, S. B., \& Touyz, S. (2014). Unique associations between young adult men's emotional functioning and their body dissatisfaction and disordered eating. Body Image, 11, 175-178. http://dx.doi.org/10.1016/j.bodyim.2013.12.002

Griffiths, S., Hay, P., Mitchison, D., Mond, J. M., McLean, S. A., Rodgers, B., Massey, R., ... Paxton, S. J. (2016). Sex differences in the relationships between body dissatisfaction, quality of life and psychological distress. Australian and New Zealand Journal of Public Health, 40(6), 518-522. http://dx.doi.org/10.1111/1753-6405.12538

Hu, L., y Bentler, P. M. (1999). Cut-off criteria for fit indexes in covariance structure analysis: Conventional criteria versus new alternatives. Structural Equation Modeling, 6(1), 1-55.

Joshi, R., Herman, C. P., y Polivy, J. (2004). Self-enhancing effects of exposure to thin-body images. International Journal of Eating Disorders, 35(3), 333-341.

Killen, J. D., Taylor, C. B., Hayward, C., Haydel, K. F., Wilson, D. M., Hammer, L., ... Strachowski, D. (1996). Weight concerns influence the development of eating disorders: A 4-year prospective study. Journal of Consulting and Clinical Psychology, 64(5), 936940. http://dx.doi.org/10.1037/0022-006X.64.5.936

Kline, R. B. (1998). Principles and practice of structural equation modeling. New York: Guilford.

Lamanna, J., Grieve, F. G., Derryberry, W. P., Hakman, M., y McClure, A. (2010). Antecedents of eating disorders and muscle dysmorphia in a non-clinical sample. Eating and Weight Disorders - Studies on Anorexia, Bulimia and Obesity, 15(1-2), 23-33.

Lavender, J. M., y Anderson, D. A. (2010). Contribution of Emotion Regulation Difficulties to Disordered Eating and Body Dissatisfaction in College Men. International Journal of Eating Disorders, 43, 352-357.

Little, T. D. (1997). Mean and covariance structures (MACS) analyses of cross-cultural data: Practical and theoretical issues. Multivariate Behavioral Research, 32, 53-76.

Monell, E., Högdahl, L., Mantilla, E. F., y Birgegård, A. (2015). Emotion dysregulation, selfimage and eating disorder symptoms in University Women. Journal of Eating Disorders, 3, 44.

Murray, S. B., Rieger, E., Karlov, L., y Touyz, S. W. (2013). An investigation of the transdiagnostic model of eating disorders in the context of muscle dysmorphia. European Eating Disorders Review, 21, 160-164. http://dx.doi.org/10.1002/erv.2194

Murray, S. B., Rieger, E., Touyz, S. W., y De la Garza Garcia, Y. (2010). Muscle dysmorphia and the DSM-V conundrum: Where does it belong? A review paper. International Journal of Eating Disorders, 43(6), 483-491.

Muthén, L. K., y Muthén, B. O. (1998-2011). Mplus User's Guide. Sixth Edition. Los Angeles, CA: Muthén \& Muthén.

Olivardia, R. (2001). Mirror, mirror on the wall, who's the largest of them all? The features and phenomenology of muscle dysmorphia. Harvard Review of Psychiatry, 9(5), 245-259.

Olivardia, R., Pope, H.G., Borowiecki, J.J., y Cohane, G.H. (2004). Biceps and body image: The relationship between muscularity and self-esteem, depression, and eating disorder symptoms. Psychology of Men and Masculinity, 5, 112-120. 
Pope, H. G. Jr., Katz, D. L., y Hudson, J. I. (1993). Anorexia nervosa and "reverse anorexia" among 108 male bodybuilders. Comprehensive Psychiatry, 34(6), 406-409.

Pope, H. G., Gruber, A. J., Choi, P., Olivardia, R., y Phillips, K. A. (1997). Muscle dysmorphia: An underrecognized form of body dysmorphic disorder. Psychosomatics, $38(6), 548-557$.

Rosenberg, M. (1965). Society and the adolescent self-image. Princeton, NJ: Princeton. University Press.

Short, J. (2005). Creating an assessment tool for muscle dysmorphia. Unpublished Masters Theses y Specialist Projects. Paper 479. Western Kentucky University, Bowlyn Green, KY.

Sim, L., y Zeman, J. (2005). Emotion regulation factors as mediators between body dissatisfaction and bulimic symptoms in early adolescent girls. The Journal of Early Adolescence, 25, 478-496.

Sim, L., y Zeman, J. (2006). The contribution of emotion regulation to body dissatisfaction and disordered eating in early adolescent girls. Journal of Youth and Adolescence, 35, 219-228.

Taylor, G. J. (2000). Recent developments in alexithymia theory and research. The Canadian Journal of Psychiatry, 45, 134-142.

Ullman, J. B. (1996). Structural equation modeling. En B. Tabachnick y L. Fidell (Eds.), Using multivariate statistics ( $3^{\mathrm{a}}$ ed., pp. 709-812). New York: HarperCollins.

Wolke, D., y Sapouna, M. (2008). Big men feeling small: Childhood bullying experience, muscle dysmorphia, and other mental health problems in bodybuilders. Psychology of Sport and Exercise, 9, 595-604. 